\title{
Chemotherapy regimen based on sorafenib combined with 5-FU on HIF-1 $\alpha$ and VEGF expression and survival in advanced gastric cancer patients
}

\author{
RONGHUI CHENG ${ }^{1}$, HONGMEI YONG ${ }^{1}$, YUNHONG XIA ${ }^{2}$, QINGSONG XIE $^{3}$, \\ GUANGYI GAO $^{1}$ and XUEYI ZHOU ${ }^{1}$
}

\begin{abstract}
${ }^{1}$ Department of Medical Oncology, The Second People's Hospital of Huaian, Huaian, Jiangsu 223002; ${ }^{2}$ Department of Medical Oncology, The Second People's Hospital of Hefei, Hefei, Anhui 230011; ${ }^{3}$ Department of General Surgery, The First Affiliated Hospital of Anhui Medical University, Hefei, Anhui 230022, P.R. China
\end{abstract}

Received August 23, 2016; Accepted January 30, 2017

DOI: $10.3892 / 01.2017 .5769$

\begin{abstract}
The present study investigated the effect of combined sorafenib chemotherapy on hypoxia-inducible factor- $1 \alpha$ (HIF-1 $\alpha)$ and vascular endothelial growth factor (VEGF) expression and survival time of patients with advanced gastric cancer. From January 2010 to December 2011, 92 patients diagnosed with advanced gastric cancer were selected and randomly divided into the treatment group and control group. The treatment group was treated with sorafenib chemotherapy combined with 5-fluorouracil (5-FU), and the control group received 5-FU. The treatment course was 3-4 cycles. During the same period, 46 healthy persons admitted to the Second People's Hospital of Huaian were selected as the controls. A volume of 3-4 ml peripheral blood from each patient and control was collected before and after treatment. The expression levels of HIF-1 $\alpha$ and VEGF in peripheral blood were measured by ELISA. The survival time of patients with advanced gastric cancer was followed and analyzed. Compared with healthy controls, serum levels of HIF-1 $\alpha$ and VEGF were significantly higher in patients with advanced gastric cancer $(\mathrm{P}<0.05)$. After chemotherapy combined with sorafenib, the peripheral blood levels of HIF-1 $\alpha$ and VEGF decreased significantly in the treatment group $(\mathrm{P}<0.05)$. The 5-year survival rate of patients in the two groups was followed. Compared with the control group, the 1-year survival rate of the treatment group was significantly higher $(\mathrm{P}<0.05)$. In conclusion, chemotherapy combined with sorafenib can effectively reduce serum levels of HIF-1 $\alpha$ and
\end{abstract}

Correspondence to: Mr. Xueyi Zhou, Department of Medical Oncology, The Second People's Hospital of Huaian, 62 Huaihai South Street, Huaian, Jiangsu 223002, P.R. China

Email: xs07nr@163.com

Key words: gastric cancer, sorafenib, hypoxia-inducible factor-1 $\alpha$, vascular endothelial growth factor
VEGF in patients with advanced gastric cancer, and improve their 1-year survival rate and prognosis. Therefore, it has significant clinical application value.

\section{Introduction}

Advanced gastric cancer is characterized by tumors that invade the muscle layer and serosa of the stomach, regardless of lesion size and occurrence of metastasis (1). The treatment strategy for advanced gastric cancer is primarily surgery, assisted by postoperative adjuvant chemotherapy (2). Because of the poor targeting ability of traditional chemotherapeutic drugs, normal tissues are also damaged during inhibition of tumor metastasis. Targeted therapies are drugs that work at the molecular level, and designed according to specific carcinogenic molecules (including proteins in tumor cells or gene fragments). These drugs specifically enter tumor cells and selectively bind carcinogenic molecules and cause tumor cell death without injuring normal tissue surrounding the tumor (3-5).

Sorafenib is a new multi-target oral drug for cancer treatment. It can be used to treat gastrointestinal stromal tumors and metastatic renal cell carcinoma for patients who are not responsive to, or cannot tolerate standard therapies (6). It can selectively target certain proteins, so as to regulate the growth and metabolism of tumor cells. Tumor cells can proliferate under hypoxic conditions. This phenomenon is closely related to the activation of hypoxia-inducible factor- $1 \alpha(\mathrm{HIF}-1 \alpha)$ and vascular endothelial growth factor (VEGF). HIF-1 $\alpha$ can enhance the metabolism of cells under hypoxic conditions and contribute to activation of VEGF to induce tumor angiogenesis (7-9).

Previous studies have found that because of the hidden incidence of gastric cancer, many patients are first diagnosed with advanced gastric cancer, or even with metastasis, and have therefore missed the optimal time for surgery $(4,10,11)$. At present, to improve the survival of patients with advanced gastric cancer, physicians apply neoadjuvant chemotherapy, which is highly effective (10). Neoadjuvant chemotherapy is the administration of 3-4 cycles of standard chemotherapy before surgery, to reduce the tumor scope and achieve the purpose of surgical resection (11). In the present study, we 
applied chemotherapy regimens based on sorafenib combined with 5-fluorouracil (5-FU) and explored their impact on the expression of VEGF and HIF-1 $\alpha$ in serum and tumor tissue.

\section{Patients and methods}

Patients. From January 2010 to December 2011, we selected 92 patients diagnosed with advanced gastric cancer by means of imaging and laboratory diagnosis. Patients were randomly divided into the treatment group and control group. Forty-six age-matched healthy controls were selected in the Second People's Hospital of Huaian during the same period.

Inclusion criteria of the treatment group. The inclusion criteria included (6): i) Diagnosis of gastric cancer that can be made by imaging, pathology, physical examination, or laboratory examination; ii) advanced gastric cancer; and iii) age over 18 years. This study was approved by the Ethics Committee of the Second People's Hospital of Huaian. Signed written informed consents were obtained from all participants before the study.

Exclusion criteria of the treatment group. The exclusion criteria included (7): i) Malignant tumors of other systems; ii) diagnosis could not be clearly made by imaging, pathology, physical examination, or laboratory examination; iii) cognitive impairment or mental illness; iv) failure to obtain patient samples; v) patients or their family members could not cooperate with the examination; vi) withdrawal of patients from the study; and vii) patients who gave up treatment or refused surgery.

Reagents. Sterile double distilled water, anti-phosphorylated VEGF (anti-p-VEGF) (1:1,000; Cell Signaling Technology, Inc., Danvers, MA, USA), $\beta$-actin antibody (1:5,000; Invitrogen Life Technologies, Carlsbad, CA, USA), phosphorylated VEGF $(1: 1,000)$, phosphorylated HIF-1 $\alpha(1: 1,000)$ and anti-phosphorylated HIF-1 $\alpha$ (1:1,000) (all from Cell Signaling Technology, Inc.), $0.9 \%$ sterile saline (Otsuka Pharmaceutical Factory, Inc., Tokushima, Japan) and TRIzol (Invitrogen Life Technologies).

Instruments. PCR amplification instrument and gel imaging instrument (both from Bio-Rad Laboratories, Inc., Hercules, CA, USA), electrophoresis apparatus (Beijing Liuyi Instrument Factory, Beijing, China), centrifuge and micro liquid transfer device (both from Eppendorf, Hamburg, Germany), Haier ice machine, Western Blot electrophoresis apparatus (Bio-Rad Laboratories, Inc.), $-80^{\circ} \mathrm{C}$ refrigerator (Thermo Fisher Scientific, Inc., Waltham, MA, USA), 5 and $10 \mathrm{ml}$ syringes (Tianjin Hanaco Medical Co., Ltd., Tianjin, China), professional experimental animals surgical instruments (Beijing Medical Equipment Factory, Beijing, China), NanoDrop 2000 spectrophotometer (Thermo Fisher Scientific, Inc.), EP tubes (Eppendorf), water bath (Beijing Medical Equipment Factory) and pathological slice machine (Leica Microsystems $\mathrm{GmbH}$, Wetzlar, Germany).

Blood collection. A total of $3 \mathrm{ml}$ venous blood was collected from the elbow vein in the morning and placed in ordinary plastic tubes. A total of $1.8 \mathrm{ml}$ was then transferred to
Table I. Analysis of general clinical data of patients in the control group and treatment group (mean $\pm \mathrm{SD}$ ).

\begin{tabular}{lcccc}
\hline Groups & $\begin{array}{c}\text { Cases } \\
(\mathrm{n})\end{array}$ & $\begin{array}{c}\text { Age } \\
\text { (years) }\end{array}$ & $\begin{array}{c}\text { BMI } \\
\left(\mathrm{kg} / \mathrm{m}^{2}\right)\end{array}$ & $\begin{array}{c}\text { Disease course } \\
(\mathrm{months})\end{array}$ \\
\hline Control & 46 & $53.8 \pm 8.4$ & $21.7 \pm 1.4$ & $9.2 \pm 1.1$ \\
Treatment & 46 & $55.2 \pm 9.2$ & $20.2 \pm 3.8$ & $10.8 \pm 2.3$ \\
T-value & - & 0.78 & 0.32 & 0.44 \\
P-value & - & 0.25 & 0.75 & 0.58 \\
\hline
\end{tabular}

anticoagulation tubes containing $0.2 \mathrm{ml} 3.8 \%$ sodium citrate. Specimens were centrifuged at $1,000 \mathrm{x}$ g for $10 \mathrm{~min}$ within $1 \mathrm{~h}$ after collection. Serum or plasma were separated and stored in $0.5 \mathrm{ml} \mathrm{EP}$ tubes after extraction, and stored at $-30^{\circ} \mathrm{C}$ until use within 1 month.

Measurement of serum VEGF and HIF-1 $\alpha$ by enzyme-linked immunosorbent assay (ELISA). VEGF and HIF-1 $\alpha$ ELISA diagnostic kits were purchased from Beijing Kangwei Century Biotech Co., Ltd. (Beijing, China) and used according to the manufacturer's instructions.

Treatment methods. We treated both groups of patients with 3-4 cycles of chemotherapy before elective surgery was performed. Treatment group: Sorafenib $(0.2 \mathrm{mg} / \mathrm{kg})+5-\mathrm{FU}$ $(0.5 \mathrm{mg} / \mathrm{kg})+$ vincristine $(1 \mathrm{mg} / \mathrm{kg})$ for 7 days comprised one course of treatment. Control group: Single 5 -FU $(0.5 \mathrm{mg} / \mathrm{kg})$ chemotherapy. Elective subtotal gastrectomy was performed after chemotherapy.

Statistical analysis. SPSS 15.0 statistical software (SPSS, Inc., Chicago, IL, USA) was used for data analysis. Measurement data are presented as mean \pm standard deviation. Comparisons between groups were by independent sample t-test. Enumeration data are presented as case or constituent ratio, and a $\chi^{2}$ test was used for comparisons. $\mathrm{P}<0.05$ was considered to indicate a statistically significant difference.

\section{Results}

Comparison of general clinical conditions of patients. We collected and analyzed the general clinical data of patients with gastric cancer in the two groups including, age, weight and course of disease. Most patients were aged from 50 to 60 years, and there were no statistically significant differences in age, body weight or course of gastric cancer among patients in the two groups $(\mathrm{P}>0.05)$ (Table I).

Measurement of serum levels of VEGF and HIF-1 $\alpha$ before and after neoadjuvant chemotherapy. VEGF and HIF-1 $\alpha$ expression levels in peripheral blood of patients and healthy controls were measured by ELISA. We found that compared with healthy controls, the serum levels of VEGF and HIF-1 $\alpha$ were significantly higher in both the treatment group and the control group before neoadjuvant chemotherapy $(\mathrm{P}<0.05)$, and no statistical differences were found between the two groups $(\mathrm{P}>0.05)$. Compared with the control group, the serum levels of VEGF 
Table II. Measurement of serum VEGF and HIF-1 $\alpha$ levels before and after neoadjuvant chemotherapy.

\begin{tabular}{|c|c|c|c|c|c|c|}
\hline Items & Groups & Cases (n) & $\begin{array}{c}\text { Before neoadjuvant } \\
\text { chemotherapy }\end{array}$ & $\begin{array}{l}\text { After neoadjuvant } \\
\text { chemotherapy }\end{array}$ & T-value & P-value \\
\hline \multirow[t]{3}{*}{ VEGF (ng/ml) } & Control & 46 & $58.8 \pm 8.4^{\mathrm{a}, \mathrm{c}}$ & $31.7 \pm 1.4$ & 4.24 & 0.02 \\
\hline & Treatment & 46 & $65.2 \pm 9.2^{\mathrm{b}}$ & $16.2 \pm 3.8^{\mathrm{d}}$ & 7.39 & 0.01 \\
\hline & Healthy control & 46 & $8.2 \pm 3.2$ & - & - & - \\
\hline \multirow[t]{3}{*}{$\mathrm{HIF}-1 \alpha(\mathrm{ng} / \mathrm{ml})$} & Control & 46 & $72.3 \pm 12.1$ & $35.3 \pm 10.6$ & 5.49 & 0.02 \\
\hline & Treatment & 46 & $78.5 \pm 16.4$ & $18.5 \pm 11.7$ & 8.39 & 0.01 \\
\hline & Healthy control & 46 & $9.2 \pm 3.4$ & - & - & - \\
\hline
\end{tabular}

Compared with the healthy control group, the serum level of VEGF was significantly higher in the control group before neoadjuvant chemotherapy, ${ }^{\mathrm{a}} \mathrm{P}=0.02$; the level of VEGF in the treatment group significantly increased, ${ }^{\mathrm{b}} \mathrm{P}=0.01$; there was no statistical difference of VEGF level between the control group and treatment group, ${ }^{\mathrm{C}} \mathrm{P}=0.39$; after neoadjuvant chemotherapy, VEGF in the treatment group significantly increased compared with control group, ${ }^{\mathrm{d}} \mathrm{P}<0.001$. VEGF, vascular endothelial growth factor; HIF-1 $\alpha$, hypoxia-inducible factor- $1 \alpha$.

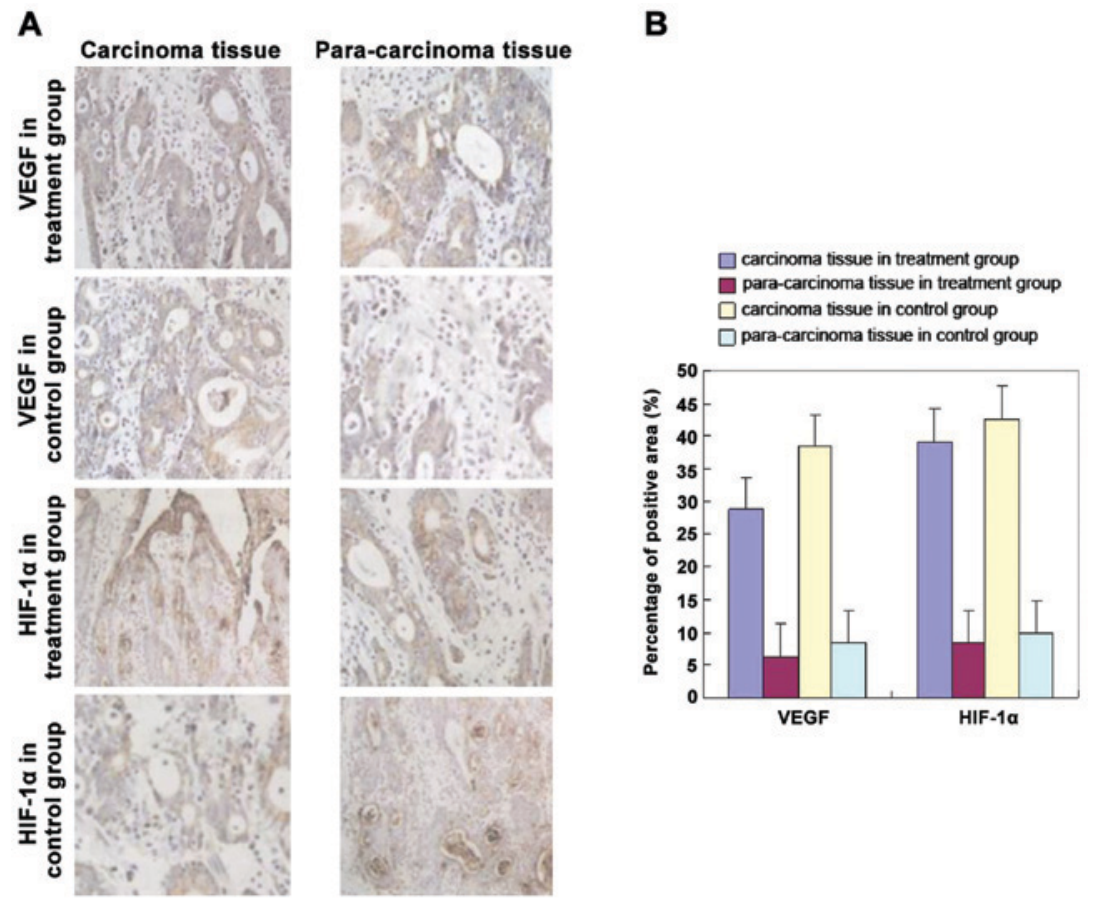

Figure 1. (A) Magnification (x400) of inverted microscopy. (B) Statistics on the percentage of positive area showed that compared with the control group, the levels of VEGF and HIF-1 $\alpha$ of tumor tissue in the treatment group were significantly lower $(\mathrm{P}<0.05)$. VEGF, vascular endothelial growth factor; HIF-1 $\alpha$, hypoxia-inducible factor-1 $\alpha$.

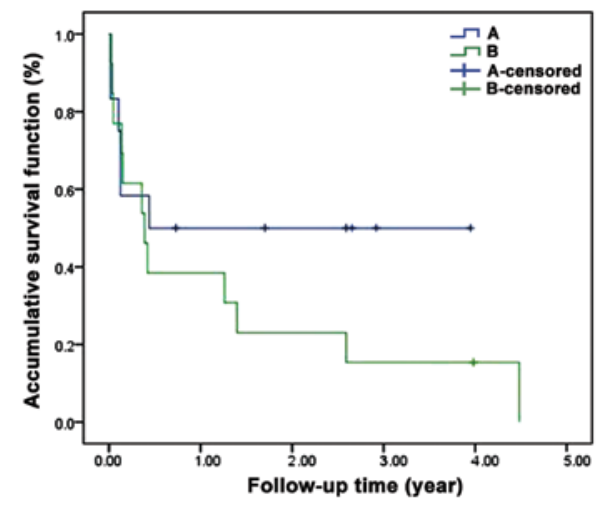

Figure 2. The 5-year survival rate of patients in the two groups. Compared with the control group, the 5-year survival rate of the treatment group was significantly higher $(\mathrm{P}<0.05)$. A, treatment group; B, control group. and HIF-1 $\alpha$ in the treatment group were significantly lower after neoadjuvant chemotherapy $(\mathrm{P}<0.05)$ (Table II).

Immunohistochemical staining of VEGF and HIF-1 $\alpha$. The levels of VEGF and HIF-1 $\alpha$ in the postoperative gastric cancer tissue of the two groups of patients were detected by immunohistochemistry. The results showed that compared with the control group, the levels of VEGF and HIF-1 $\alpha$ in the treatment group were significantly lower (Fig. 1) $(\mathrm{P}<0.05)$.

Survival curve. The 5-year survival rate of patients in the two groups were followed. Compared with the control group, the 5 -year survival rate of the treatment group was significantly higher $(\mathrm{P}<0.05)$ (Fig. 2). 


\section{Discussion}

Gastric cancer has one of the highest mortality rates in cancer. Increasing numbers of patients with advanced gastric cancer achieve good therapeutic effects because of several recent advances in treatment and the development of targeted therapeutic agents. However, because of hidden clinical symptoms, patients are usually first diagnosed with advanced gastric cancer, with poor prognosis and the median overall survival period is $<12$ months (12). Therefore, prolonging survival time and improving survival rate of patients with advanced gastric cancer are key to treatment (13).

Angiogenesis is the basis of proliferation, invasion and metastasis of advanced gastric cancer. VEGF can induce the proliferation of vascular endothelial cells, promote endothelial cell survival of the newly formed vessels, and increase vascular permeability (5), which contribute to metastasis and invasion of tumor cells. Our results showed that serum levels of VEGF and HIF-1 $\alpha$ were both significantly increased in advanced gastric cancer patients $(\mathrm{P}<0.05)$. These observations were consistent with previous studies $(5,14)$. We believe that 5-FU combined with sorafenib can significantly reduce the expression of VEGF in gastric cancer tissue. Therefore, drugs targeting VEGF and its receptor will ideally help to suppress tumor proliferation, invasion and metastasis. In addition, a previous study illustrated that there is a phenomenon in most cancer cells known as the 'Warburg effect', which refers to tumor cells in hypoxic or anoxic conditions that can proliferate by increasing the rates of glycolysis and lactic acid fermentation (15). The rates of glycolysis and lactic acid fermentation of tumor cells can also increase significantly under normoxic conditions. Hypoxia is a common feature of solid tumors in the middle and late stage. Hypoxia will stimulate a series of adaptive responses such as tumor angiogenesis, increased drug resistance and enhanced metastasis ability (16). The transcription factor, HIF-1 $\alpha$, plays a central role in these processes. The expression of HIF-1 $\alpha$ can increase significantly under hypoxic conditions, and it improves the cell's tolerance to hypoxia. It has therefore become a potential anticancer target as well (17). Our study showed that in addition to the increase of serum VEGF in patients with advanced gastric cancer, HIF-1 $\alpha$ level was also significantly higher than in healthy controls. Immunohistochemical staining of tumor tissue showed that HIF-1 $\alpha$ level in tumor tissue was significantly increased compared with adjacent normal tissue. This demonstrates that HIF-1 $\alpha$ overexpression occurs in advanced gastric tumor tissue. We believe that increased HIF-1 $\alpha$ expression level can further enhance tumor tissue survival in the hypoxic environment and promote anaerobic metabolism of tumor tissue. Yu et al (18) found that in this process, the transcription factor c-Jun blocked proteasome-mediated degradation of HIF- $1 \alpha$ and stabilized HIF-1 $\alpha$ protein. In addition, c-Jun reduced HIF- $1 \alpha$ ubiquitylation and increased its stability, which resulted in enhanced VEGF transcription and increased VEGF secretion by tumor cells, which promoted angiogenesis. The above results showed that c-Jun exerted a molecular barrier function by binding to the ODD domain of HIF-1 $\alpha$, prevented its ubiquitination and degradation, stabilized HIF-1 $\alpha$ protein, and consequently promoted transcription and mediation of tumor angiogenesis. Sorafenib is a new oral multi-target drug for cancer treatment.
The primary goal during its development was to treat gastrointestinal stromal tumors and metastatic renal cell carcinoma for patients who are not responsive to, or cannot tolerate standard therapies. It selectively targets certain protein receptors which act as molecular switches in the process of tumor growth. Previously, drug monotherapy was commonly used for the treatment of hepatocellular carcinoma (19), renal clear cell carcinoma (20), and colorectal cancer (21) and good therapeutic effects were obtained. Through the evaluation of 5 -year survival rate among patients in the 2 groups, we found that when chemotherapy based on sorafenib combined with 5-FU was used, the postoperative 5-year survival rate was significantly increased, thus demonstrating that this combination chemotherapy has clinical value.

In conclusion, we believe that VEGF and HIF-1 $\alpha$ have important clinical value for the evaluation of proliferation and metastasis of advanced gastric cancer patients. When neoadjuvant chemotherapy is employed and its clinical efficacy is observed, VEGF and HIF-1 $\alpha$ can be used as new observational indices of the efficacy of neoadjuvant chemotherapy.

\section{References}

1. Yang XW, Gao F, Chen YJ and Teng FM: The clinical study of urokinase-type plasminogen activator and vascular endothelial growth factor in gastric cancer. Cell Biochem Biophys 72: 649-652, 2015.

2. Chang Y, Niu W, Lian PL, Wang XQ, Meng ZX, Liu Y and Zhao R: Endocan-expressing microvessel density as a prognostic factor for survival in human gastric cancer. World $\mathbf{J}$ Gastroenterol 22: 5422-5429, 2016.

3. Liu N, Ding D, Hao W, Yang F, Wu X, Wang M, Xu X, Ju Z, Liu JP, Song Z, et al: hTERT promotes tumor angiogenesis by activating VEGF via interactions with the Sp1 transcription factor. Nucleic Acids Res: Jun 20, 2016 (Epub ahead of print).

4. Jun KH, Lee JE, Kim SH, Jung JH, Choi HJ, Kim YI, Chin HM and Yang SH: Clinicopathological significance of N-cadherin and VEGF in advanced gastric cancer brain metastasis and the effects of metformin in preclinical models. Oncol Rep 34: 2047-2053, 2015.

5. Wang L, Zhou R, Zhao Y, Dong S, Zhang J, Luo Y, Huang N, Shi M, Bin J, Liao Y, et al: MACC-1 promotes endothelium-dependent angiogenesis in gastric cancer by activating TWIST1/VEGF-A signal pathway. PLoS One 11: e0157137, 2016.

6. Gasser M and Waaga-Gasser AM: Therapeutic antibodies in cancer therapy. Adv Exp Med Biol 917: 95-120, 2016.

7. Tong W, Ye F, He L, Cui L, Cui M, Hu Y, Li W, Jiang J, Zhang DY and Suo J: Serum biomarker panels for diagnosis of gastric cancer. Onco Targets Ther 9: 2455-2463, 2016.

8. Liu N, Zhou N, Chai N, Liu X, Jiang H, Wu Q and Li Q: Helicobacter pylori promotes angiogenesis depending on Wnt/beta-catenin-mediated vascular endothelial growth factor via the cyclooxygenase- 2 pathway in gastric cancer. BMC Cancer 16: 321, 2016.

9. Siegel R, Ma J, Zou Z and Jemal A: Cancer statistics, 2014. CA Cancer J Clin 64: 9-29, 2014.

10. Qu J and Qu X: The predictors of response to neoadjuvant chemotherapy in patients with locally advanced gastric cancer. Cancer Biomark 17: 49-54, 2016.

11. Samalin E and Ychou M: Neoadjuvant therapy for gastroesophageal adenocarcinoma. World J Clin Oncol 7:284-292, 2016.

12. Jemal A, Bray F, Center MM, Ferlay J, Ward E and Forman D: Global cancer statistics. CA Cancer J Clin 61: 69-90, 2011.

13. Ferlay J, Shin HR, Bray F, Forman D, Mathers C and Parkin DM: Estimates of worldwide burden of cancer in 2008: GLOBOCAN 2008. Int J Cancer 127: 2893-2917, 2010.

14. Fourgeaud C, Derieux S, Mirshahi S, Besbes S, Chidiac J, Mahé I, Contant G, Pocard M, Soria J and Mirshahi M: PO-23 - expression of heparanase in cancer as biomarker of malignancies: overexpression in an aggressive, poor survival gastric cancer 'gastric signet ring cell carcinoma' compared with that of other gastric cancers. Thromb Res 140 (Suppl 1): S184-S185, 2016. 
15. Scott DA, Richardson AD, Filipp FV, Knutzen CA, Chiang GG, Ronai ZA, Osterman AL and Smith JW: Comparative metabolic flux profiling of melanoma cell lines: beyond the Warburg effect. J Biol Chem 286: 42626-42634, 2011.

16. Shi K, Ziegler SI and Vaupel P: Molecular imaging of tumor hypoxia: existing problems and their potential model-based solutions. Adv Exp Med Biol 923: 87-93, 2016.

17. Guimarães TA, Farias LC, Santos ES, de Carvalho Fraga CA, Orsini LA, de Freitas Teles L, Feltenberger JD, de Jesus SF de Souza MG, Santos SH, et al: Metformin increases PDH and suppresses HIF-1 $\alpha$ under hypoxic conditions and induces cell death in oral squamous cell carcinoma. Oncotarget: Jul 26, 2016 (Epub ahead of print).

18. Yu B, Miao ZH, Jiang Y, Li MH, Yang N, Li T and Ding J: c-Jun protects hypoxia-inducible factor-1alpha from degradation via its oxygen-dependent degradation domain in a nontranscriptional manner. Cancer Res 69: 7704-7712, 2009.
19. Borentain P, Gregoire E, Louis G and Gerolami R: Successful liver transplantation for hepatocellular carcinoma following down-staging using sorafenib single therapy. Liver Int 36: 1393, 2016.

20. Rini BI, Escudier B, Tomczak P, Kaprin A, Szczylik C, Hutson TE, Michaelson MD, Gorbunova VA, Gore ME, Rusakov IG, et al: Comparative effectiveness of axitinib versus sorafenib in advanced renal cell carcinoma (AXIS): a randomised phase 3 trial. Lancet 378: 1931-1939, 2011.

21. Martchenko K, Schmidtmann I, Thomaidis T, Thole V, Galle PR, Becker M, Möhler M, Wehler TC and Schimanski CC: Last line therapy with sorafenib in colorectal cancer: a retrospective analysis. World J Gastroenterol 22: 5400-5405, 2016. 